\title{
ANDRÉS BELLO Y JOSÉ VICTORINO LASTARRIA: LA APROPIACIÓN LATINOAMERICANA DEL ROMANTICISMO Y LA ILUSTRACIÓN
}

Mg. Stefan Vrsalovic M.*

El siguiente artículo tiene como propósito indagar en las bases filosóficas que existen en la llamada "discusión historiográfica" entre Andrés Bello y José Victorino Lastarria. Básicamente, plantea la plausibilidad de sostener que en el siglo XIX hubo en Chile una apropiación de las ideas filosóficas europeas que permite establecer los inicios de una filosofía propiamente chilena.

Palabras clave: Apropiación, reproducción, Ilustración, Romanticismo, Latinoamérica.

\section{ANDRÉS BELLO AND JOSÉ VICTORINO LASTARRIA: THE LATIN- AMERICAN APPROPRIATION OF ROMANTICISM AND ILLUSTRATION}

The following article purports to inquire in the philosophical bases that are found in the so called "historiographical discussion" between Andrés Bello and José Victorino Lastarria. Basically it raises the acceptability of sustaining that there was an appropriation of the European philosophical ideas in Chile during the 19th century, that allows to establish the beginnings of a properly chilean philosophy.

Keywords: Appropriation, reproduction, Enlightenment, Romanticism, Latin America.

*Universidad de Chile, Santiago, Chile. Correo electrónico: stefanvrs@gmail.com 



\section{Entre la Reproducción y la Apropiación}

La relación entre Andrés Bello y José Victorino Lastarria puede verse desde dos aristas no excluyentes: como maestro y discípulo, y como contrincantes intelectuales. Desde esta segunda perspectiva es que el presente artículo presenta a ambos autores, basándose específicamente en la disputa historiográfica que se efectúo entre 1844 y $1848^{1}$. A partir de esta discusión queremos sostener que hubo una apropiación latinoamericana del pensamiento ilustrado y romántico y señalar que fue una de las primeras discusiones en las que se puede rastrear una cierta recepción crítica de las ideas europeas.

Básicamente, la disputa se centró en cómo ver el pasado y su función. Bello defendió, desde una matriz más bien romántica, una historia narrativa que debía contar los hechos sin mistificarlos con teorías. Lastarria, en cambio, sostuvo una filosofía de la historia, desde ciertos conceptos universales propios de la Ilustración, como razón, progreso y modernidad. Pensó que en Chile aún se prolongaba un modo de ser colonial que debía ser superado, que la independencia no era, cabalmente, lo que la voz oficial sostenía. Leopoldo Zea en su texto El Pensamiento Latinoamericano cataloga a Lastarria, por esta posición, dentro de los "emancipadores mentales" del siglo XIX hispanoamericano.

La interpretación que se ha establecido de esta disputa es que Bello defiende una historia desde una mirada romántica, preocupándose de la Nación en particular y negando la validez de ideas universales (ilustradas en este caso) y Lastarria adscribe claramente a una postura ilustrada. En consecuencia, lo que hizo cada uno fue reproducir un modo de conceptualizar la historia articulado en Europa. Pero, ¿fue sólo reproducción?, o ¿hubo algo de apropiación, aun cuando no fuera al nivel de la "gran” filosofía europea? ¿La relación, por ejemplo, que Lastarria establece entre instituciones y costumbres ${ }^{2}$ no es una forma nueva de articular el pensamiento ilustrado?

1 No es el propósito del artículo ahondar en esta disputa y explicarla. Para ello ya existen varios textos que la ilustran de buena manera, por ejemplo: Subercaseaux, Bernardo, Cultura y sociedad liberal en el siglo XIX (Lastarria, ideología y literatura), Editorial Aconcagua, Chile, 1981; Stuven, Ana María, La Seducción de un Orden: las elites y la construcción de Chile en las polémicas culturales y politicas del siglo XIX, Ediciones Universidad Católica de Chile, Santiago, 2000; Oyarzún, Luis, El Pensamiento de Lastarria, Editorial Jurídica de Chile, Valparaíso, 1953; JAKSIC, Iván, Andrés Bello. La pasión por el Orden, Editorial Universitaria, Santiago, 2001; Illanes, María Angélica, Chile des-centrado: Formación socio-cultural republicana y transición capitalista (1810 - 1910), Lom, Santiago, 2003; Colmenares, Germán, Las Convenciones contra la cultura: ensayo sobre la historiografía hispanoamericana del siglo XIX, Centro de Investigaciones Diego Barros Arana, Santiago, 2006.

$2 C f r$. Lastarria, José Victorino, "Investigaciones sobre la Influencia social de la conquista i del sistema colonial de los españoles en Chile", en Miscelánea Histórica i Literaria, Tomo I, Imprenta "La Patria", Valparaíso, 1868, pp. 3-138. 
Antes de hacernos cargo de lo que estas preguntas implican, revisaremos brevemente tres posturas significativas que, a este respecto, han definido la filosofía latinoamericana como una imitación ${ }^{3}$. Es el caso de Manfredo Kempff, que en su texto Historia de la filosofía latinoamericana de 1958, sostiene que el problema filosófico en nuestro continente refleja una dinámica caracterizada por una actitud pasiva, receptora y acrítica, que sigue los "mandamientos" de la cuna de la cultura. Lo que aparece es:

\begin{abstract}
Europa dando las orientaciones y las direcciones y nosotros asimilándolas e incorporándolas a nuestro panorama cultural. Siempre Europa nos ha encontrado en actitud de adaptación y aprendizaje. Estamos colocados frente a ella como un alumno que repite los movimientos de su profesor de gimnasia ${ }^{4}$.
\end{abstract}

En una línea parecida, Salazar Bondy es incluso más categórico en su texto ¿Existe una filosofía de nuestra América? del año 1968: "el desenvolvimiento ideológico hispanoamericano corre paralelo con el proceso del pensamiento europeo y los cambios que se producen en él coinciden estrechamente con las transformaciones de la filosofía occiden-

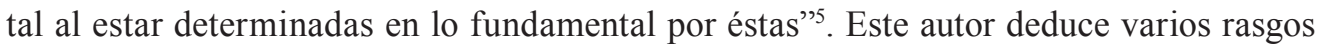
negativos del pensamiento latinoamericano como el sentido imitativo de la reflexión, la acentuada disposición receptiva, la superficialidad y pobreza teórica, la ausencia de un método propio, cierta frustración intelectual y una marcada distancia entre el pensador y la sociedad. Para Salazar Bondy, por lo tanto, no habría más que una estéril reproducción que sólo tiene cambios en virtud de fines prácticos: "las filosofías trasplantadas resultaron sujetas a cambios y recortes y aplicaciones, con vistas a un uso práctico. Fueron así insertadas en el contexto local y utilizadas dentro de ciertos límites como elementos apropiados para enfrentar los problemas de la realidad"6. Pero el problema radica en que la solución sería hacer de los rasgos negativos, positivos, es decir, que el pensador latinoamericano sea original, profundo y riguroso, que tenga una metodología que lo caracterice, etc. Sin embargo, para poder establecer aquello, es necesaria la comparación, es decir, ¿frente a quién se puede afirmar que el pensador es original?, la única respuesta es frente al europeo. La solución de Bondy es, en definitiva, crear un dualismo abismal entre el "nosotros" y el "ellos".

Siguiendo una línea parecida, Iván Jaksic y Jorge Gracia sostienen en su texto Filosofía e identidad cultural en América Latina de 1988 que habría tres posturas para responder a la pregunta ¿existe o no una filosofía latinoamericana?, la universalista, culturista y crítica. Cada postura respondería de forma tanto negativa como positiva a la pregunta

3 No son las únicas posturas que se han articulado en el siglo XX, pero ilustran y fundamentan la conclusión a la que se quiere llegar en el presente artículo.

4 Kempf, Manfredo, Historia de la filosofia latinoamericana, Zig-Zag, Santiago, 1958, p. 30.

5 Bondy, Augusto Salazar, ¿Existe una filosofía de nuestra América?, Siglo Veintiuno, México, 1969, p. 36.

$6 \quad$ Ibid., p. 38. 
planteada. Dependiendo a cuál sentido uno se inscriba dependerá si la respuesta es afirmativa o negativa. Por ejemplo, el que crea que la ideología, entendida como la filosofía para sustentar ciertos puntos de vista político-sociales, y por ende, no como una empresa libre, abarcó todo desarrollo del pensamiento latinoamericano, sostendría la no-existencia de una filosofía latinoamericana. Por el contrario, para el que sostenga que la ideología no impermeabilizó todo el desarrollo filosófico, si existiría. En conclusión, depende de la postura del observador en cuestión, o en otras palabras, la pregunta se responde con un "aún no se sabe, es relativo al sujeto pensante en cuestión".

Estas tres posturas, brevemente planteadas, han marcado, en rasgos generales, la forma de concebir lo que se puede llamar el pensamiento latinoamericano. Esas tres posturas entran en lo que se ha denominado modelo de reproducción, esto quiere decir, que simplemente se importan ideas de las culturas civilizadas, por parte de las elites, tratando de aplicarlas como esquemas absolutos generando un evidente desfase entre el esquema teórico y la especificidad de la realidad histórico-cultural que se explica.

Bernardo Subercaseaux en un artículo llamado, precisamente, Reproducción y Apropiación, sostiene que "el desfase se produciría porque ciertas corrientes de pensamiento que surgen en Europa de condiciones históricas específicas y concretas, empiezan a existir en América Latina sin que las circunstancias y hechos que las generaron logren todavía una presencia o una fuerza suficiente" ". Subercaseaux da como ejemplo, el liberalismo sin burguesía o el barroco sin reforma. Se deduce, entonces, que las elites articularon su pensamiento reproduciendo el pensamiento extranjero para vincularse con lo que entendían que era la civilización y modernización, pero, según el profesor Subercaseaux, siempre desde un modelo de reproducción.

Por otra parte, la apropiación sería el proceso creativo con el cual se convierten en propios los elementos que son ajenos a la cultura latinoamericana:

\begin{abstract}
A los conceptos unívocos de "influencia", "circulación” o "instalación" (de ideas, tendencias o estilos) y al supuesto de una recepción pasiva e inerte, se opone, entonces el concepto de "apropiación", que implica adaptación, transformación o recepción activa en base a un código distinto y propio $^{8}$.
\end{abstract}

En este sentido, rompe de cierta manera el dualismo que establece la reproducción, el ellos versus el nosotros como sustancialmente distintos; por ejemplo, este modelo generaría un "liberalismo latinoamericano", en cambio, el de reproducción, un "Liberalismo en Latinoamérica".

7 Subercaseaux, Bernardo, "Reproducción y Apropiación: dos modelos para enfocar el diálogo intercultural”, en Dialogosfelafacs.net. http://www.dialogosfelafacs.net/dialogos epoca/pdf/23-BernardoSubercaseaux.pdf, p. 1. 8 Ibid., p. 3. 
La apropiación se refiere, justamente, al movimiento entre lo idéntico y diferente de cada cultura. Subercaseaux concluye que el modelo de reproducción ha dominado en gran parte del desarrollo cultural en América Latina, y que recién se está efectuando un proceso de apropiación. Pero ¿la relación que establece Lastarria entre premisas ilustradas y románticas (por ejemplo, la relación entre instituciones y costumbres), no tiene como consecuencia un pensamiento propio y determinado por, justamente, las condiciones socio-culturales del país? Es desde está relación que establece Lastarria al interior de su pensamiento, que se puede mostrar que ciertas posturas respecto al pensamiento latinoamericano han sido unilaterales y se han dejado llevar por las posturas europeas o, como dice Herder en Ideas para una filosofía de la historia universal, que "si partimos de que la noción de la cultura se define por la cultura europea no hay que extrañarse de que la

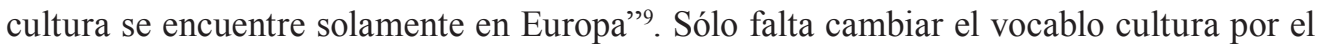
de filosofía.

Sin embargo, en la otra calzada y refutando tal tradición que se ha ido debilitando a través de los tiempos, podemos encontrar, entre otros, a la profesora Carla Cordua y al profesor José Santos.

La profesora Carla Cordua, a pesar de no ser una especialista en los temas relacionados con Latinoamérica, plantea, en su texto Incursiones, una hipótesis que resalta la necesariedad de la imitación (que vendría a ser sinónimo de reproducción) a la hora de un proceso de creación propia, es decir, de una apropiación de las ideas por parte de los pensadores latinoamericanos. La profesora señala que el problema radica en la errada diferenciación entre originalidad e imitación, "pero todo lo que se aprende procede la imitación"10 desde el lenguaje hasta las complejas formulas de la física cuántica. En cambio, la mera imitación no es formal, sino que se llena de contenido al realizarse en un contexto social, cultural y político distinto al europeo, por ello, la profesora sostiene que:

\section{[...] los resultados de nuestras repeticiones no son en ninguna medida europeos, sino hispanoamericanos de punta a cabo. [...] Las llamadas "influencias" no son fuerzas ajenas que nos moldean fatalmente. [...] No hay influencias como tales y que actúen por sí solas; ya el acogerlas en vez de rechazarlas inicia el proceso de su apropiación, que no es nunca neutro, sino que las transforma, las adapta, las tuerce y reconfigura ${ }^{11}$.}

De esta manera, para Carla Cordua, "nuestras pequeñas originalidades presuponen las innumerables maneras de repetir las cosas humanas a las que accedemos mediante la imitación de otros, de los que nos precedieron y estuvieron disponibles para tomarnos de

9 Herder, Johann Gottfried, Ideas para una filosofía de la historia de la humanidad, Losada, Buenos Aires, 1959, p. 262.

10 Cordua, Carla, Incursiones, Ediciones Universidad Diego Portales, Santiago, 2007, pp. 266-267.

11 Ibid., pp. 273-274. 
la mano y enseñarnos a caminar erectos"12. El intelectual latinoamericano trata de rehusar la idea de la imitación o mera reproducción debido a que significaría, según el entendimiento común, la dependencia de la cultura europea y no la autonomía ni, menos, una real originalidad.

Escuchando a nuestros pensadores: Bello, no sólo defendió una historia narrativa que responde a un corte más romántico que ilustrado, sino que sostuvo en su Modo de estudiar la historia que la naturaleza del hombre es dominar al otro, ¿cómo entender aquella mixtura desde sólo un modelo de reproducción? Lastarria, por otra parte, también es un claro ejemplo de la postura que se trata de sostener desde la profesora Cordua, cuando señaló tanto en sus Investigaciones... como en su Bosquejo... que las instituciones tienen que ser expresión de las costumbres de cada pueblo particular; idea planteada recién con Herder.

En virtud de la reproducción de diversas ideas se fue articulando un pensamiento que es propio y que se funde en matices irrepetibles e inimitables. Si se analizara a un pensador desde el modelo de reproducción, el análisis se definiría en las problemáticas europeas, dejando a Latinoamérica como producto de una comparación. En cambio, si se parte desde las problemáticas particulares del continente, en específico, el chileno, se entenderá que las ideas no fueron sólo reproducidas, sino apropiadas debido a la finalidad que éstas perseguían, es decir, el objetivo que los intelectuales del siglo XIX trataron de resolver a través de las corrientes europeas fueron específicos de Chile. No ocurre en Europa un proceso de independencia político, que para algunos no será completo hasta que se realice como independencia social-cultural. Ese proceso, que había que completar, de forma radical para unos y de forma moderada para otros, marcó la pauta intelectual de comienzos del siglo XIX.

Esta apropiación originada por la reproducción es bastante clara en Lastarria. Reproduciendo la filosofía de la historia ilustrada se encontró con una realidad a la cual no podía responder desde esa matriz, por ello, se hizo de ciertas premisas románticas, como fue la idea de la correspondencia entre costumbres e instituciones, lo que le permitió tomar una postura crítica frente a la realidad concreta. Por lo tanto, para responder a las problemáticas específicamente chilenas tuvo que relacionar dos corrientes, es decir, de la reproducción de ambas corrientes se generó una síntesis que tiene como resultado una apropiación del pensamiento europeo ya que es original y presentó colores propios determinados por las condiciones socio-culturales particulares.

En definitiva, el modo de apropiación parte desde la base que la realidad concreta ${ }^{13}$ en la que se mueven los pensadores latinoamericanos es distinta a la europea, o siguiendo a

12 Ibid., p. 275.

13 La problemática moderna que versa sobre un sujeto onto-creador (la conciencia de Hegel, por ejemplo) o configurador (tiempo, espacio y categoría de Kant) supera esta discusión. A pesar que se puede criticar la validez teórica de algo así como una "realidad concreta", el propósito es apuntar a que cada pensador es hijo 
lo que dice el Prof. José Santos en su texto Conflicto de representaciones responden más a la representación de "Nuestra América" que de la representación del "Nuevo mundo". La apropiación intelectual no fue un mero aplicar de forma abstracta, esto quiere decir, sin importar las condiciones particulares, sino que fue a partir de éstas que se articuló el pensamiento y nació, aunque fuesen simples, nuevas formas de conceptualizar y pensar la realidad tanto latinoamericana como chilena.

Recalcando este punto, podemos decir que si sostuviésemos que Andrés Bello sólo reprodujo ideas europeas, no se podría explicar por qué, siendo partidario de un gobierno conservador, crea, junto a otros intelectuales y políticos, la Universidad de Chile que viene a expresar el primer gran paso de la tradición a la modernidad. Esto no niega el elemento conservador (existe una facultad de teología que es compuesta por los miembros de la antigua universidad de San Felipe), ni tampoco lo lleva a ser sólo un pensador ilustrado, sino que obliga a analizarlo desde las mismas condiciones desde donde articuló su modo de ver el mundo.

El punto de partida, la "Nuestra América" es la que permite entender como diversas voces interactuaron e interactúan en un mismo pensamiento, voces que en Europa se encontraban irreconciliablemente opuestas. De allí la complejidad, por ejemplo, que siempre se le ha adjudicado a la persona de Bello. El mayor problema era comprender cómo ejercía diversas actividades y desde diversos puntos de vista, pero tal complejidad se resuelve en parte al dejar de analizarlo desde los ojos europeos, al dejar de pensar de forma eurocentrista.

No obstante, cabe señalar que la mera reproducción, como modelo pasivo - receptivo, tendría como consecuencia la facilidad de encasillar a los pensadores latinoamericanos bajo los movimientos intelectuales de la misma manera como se realiza con los europeos. A pesar de las leves diferenciaciones, nadie está en contra de denominar a Voltaire como Ilustrado, al igual que Montesquieu, y como romántico a Herder o Hölderlin. Pero en Latinoamérica, y en Chile, tales categorías no responden y no expresan al pensador en toda su amplitud. Como se matizó brevemente, Lastarria puede ser preponderantemente ilustrado, pero no deja de articular ideas desde una matriz romántica, por ello, es inviable catalogarlo como puramente ilustrado ya que sería, en definitiva juzgar al pensador sólo desde las categorías europeas. Muestra de esta diferencia son, por ejemplo, las opiniones de Subercaseaux y Colmenares; el primero, sostiene que Lastarria instauró la corriente romántica, al menos en su aspecto literario, en Chile; en cambio, el segundo, sostiene que Lastarria es estrictamente ilustrado lo que tiene como consecuencia la articulación

de su tiempo y, por lo tanto, es con su presente con el cual se enfrenta y, es éste el que conceptualizan. Sus problemáticas, específicamente la disputa historiográfica, se alimentó de la praxis. El pensamiento, en este sentido, se articuló en virtud de un problema que estuvo arraigado en la realidad (político-fundacional), de allí que se sostendrá que el desarrollo no fue por el pensamiento en sí mismo, sino que fue por lo que pudo o no pudo responder de la realidad. 
de una filosofía de la historia a-histórica. Estas dos formas de explicar el pensamiento de Lastarria, a pesar de no ser excluyentes la una de la otra, expresan la europeización que se ha llevado a cabo en la mayoría de los análisis sobre el pensamiento latinoamericano.

Por ello, el cambio metodológico que se ha propuesto implícitamente en esta artículo, permite afirmar que en el siglo XIX no sólo se llevó a cabo un proceso de recepción y reproducción, sino, además, las condiciones socio-culturales particulares, permitieron y me atrevo a sostener, exigieron, que se formase un pensamiento en base a una, si se quiere leve pero verificable, apropiación de las ideas filosóficas europeas.

El modelo de apropiación anteriormente descrito permite responder y comprender de mejor manera a nuestros pensadores. Analizarlos sólo desde una mera reproducción plantea una pasividad que no se hace cargo de la real vida intelectual a la que contribuyeron con su trabajo.

\section{Apropiación: tensión entre filosofía y política}

Ahora bien, la señalada apropiación que se estableció en el anterior apartado, ¿se realizó por el desarrollo de la filosofía misma, por las relaciones de ideas que cada autor articuló respondiendo a una razón ontológica, o, más bien, tales relaciones se realizaron por un objetivo político? Es inviable pensar la filosofía, en el siglo XIX chileno, como una disciplina independiente y autónoma de otras, sino más bien, su orientación estuvo marcada siempre por la praxis y estuvo orientada por un objetivo que se puede llamar político-fundacional, especifico de Latinoamérica.

Cabe señalar que es clara la marcada dicotomía que se establece acá entre razón filosófica y criterio político ${ }^{14}$ como si ambos modos fuesen excluyentes entre sí. La historia del pensamiento demuestra que no lo son, de allí una amplia tradición en lo que se ha denominado filosofía política, pero sin perjuicio de ello, es importante destacar que, generalmente, un modo tiene más peso que el otro, es decir, que no están en igualdad de condiciones. Por ejemplo, Aristóteles articula una filosofía política subsumida a su ontología, su concepción estuvo determinada por una comprensión ontológica del hombre, de la que deriva su concepto de animal político y que explica la dificultad de interpretar su idea de la condición política sin vincularla con la Metafísica.

Sin embargo, lo que se trata de sostener es que el criterio político relacionado a la fundación de una Nación, tuvo más peso a la hora de articular un pensamiento que una

14 Considerando las diversas formas de comprender el concepto de política y también de lo político, se tomó como concepto orientador la política en virtud de una teoría del Estado, pero moldeándolo al propio contexto sociocultural que se estudia, esto quiere decir, procesos que fueron específicos de Latinoamérica como: la Independencia (política y social-mental), la relación con el pasado (educador o legitimador), su vínculo con la idea de civilización y modernidad, la importación, recepción, reproducción y apropiación de las ideas europeas, etc. 
razón filosófica, esto debido, justamente, al momento en que estuvieron inmersos ambos autores. No es el modo cómo comprendieron al hombre lo que hizo a Lastarria tratar de fundar una Nación de corte liberal ni a Bello de corte conservador, si no, por el contrario, fue por su postura respecto a lo político que derivaron su forma de conceptualizar al hombre, la historia, la libertad, etc.

Siguiendo esta línea, Luis Oyarzún en El Pensamiento de Lastarria, sostiene que lo orientador fue la política y que en ello se centró la disputa, "la discusión no era, pues, puramente académica: era, en el fondo, política. Pero los diferentes planos que la cuestión implicaba no fueron nítidamente discernidos" ${ }^{15}$, sin embargo, no es del todo claro que sólo fue un planteamiento político, entendido hasta el momento abstractamente como una forma de ordenar la realidad y marcar cómo debe ser ésta, el criterio con el cual ambos pensadores articularon sus ideas.

Lo que si se plantea es la fuerte inclinación a la praxis. Toda idea, si no tiene un propósito en la praxis queda relegada. Es por ello que "Lastarria consideraba que Bello, -sostiene Oyarzún-, a semejanza de Cousin, su maestro de entonces, actuaba movido por la intención de defender las tendencias políticas dominantes que se oponían a la historia concebida como crítica del pretérito y como arma de combate ideológico"16. La importancia de la disputa radicó, en buena medida, en la funcionalidad de la historia, y ésta tuvo su importancia por las consecuencias que pudo tener en el ámbito político, es decir, no en su forma abstracta señalada en el párrafo anterior, sino, en su expresión concreta como modo de fundar una nación en relación con ciertas matrices culturales propias.

En resumidas cuentas, el objetivo de gran parte de los intelectuales de Latinoamérica en el siglo XIX fue articular una forma de Nación, pero no en un sentido formal, sino concreto, conservadora o liberal generalmente, bajo ciertas ideas importadas de Europa. La lucha, en este sentido, discurrió respecto al Estado que cada pensador creyó que debió establecerse para el desarrollo de la nación creciente. Es por ello, que el criterio político se relaciona con la pregunta cómo debe ser el Estado de la nueva nación, y por lo tanto, el trasfondo político que estuvo subsumido en la disputa, en primera instancia, vino a ser la teoría del Estado que trató de responder y dar cuenta de quién, cómo y para quién se gobierna.

Esta manera de comprender la política permite explicar el por qué tanto Lastarria como Andrés Bello articularon su pensamiento en base a teorías que estuvieron en contradicción en Europa, a saber, Ilustración y Romanticismo. La necesidad que obligó a estos autores a reproducir y apropiarse de ciertas premisas tanto ilustradas como románticas (en el caso de Bello, eclécticas en última instancia) se derivó del fin político que se buscó, el

15 Oyarzún, Luis, El Pensamiento de Lastarria, Editorial Jurídica de Chile, Valparaíso, 1953, p. 215.

16 Ibid., p. 210. 
tipo de Estado que se quiso establecer, y, en definitiva, de la realidad inmediata en la que estuvieron pensando y actuando.

Sin embargo, no basta con afirmar que el concepto de política que se utilizó para analizar tanto a Lastarria como a Bello tiene relación con la teoría del Estado ya que se estaría cometiendo la falta que se ha tratado de evitar desde el comienzo, aplicar teorías preexistentes al contexto latinoamericano y, en definitiva, hipostasiar la misma particularidad latinoamericana. El criterio político de ambos autores estuvo empañado por sus mismos procesos y condiciones socio-culturales. La disputa, en estricto rigor, no fue por qué tipo de Estado debió gobernar, sino, de allí la particularidad de Latinoamérica, qué tipo de nación se debió desarrollar. Las preguntas tales como ¿esta nueva nación debe ser una continuación de la Colonia o debe cortar radicalmente con ella?, ¿cómo y cuál debe ser la conceptualización que se debe hacer del pasado?, ¿qué ideas se deben instaurarán para crear y sostener esta nueva nación?, ¿cuál debe ser la intelectualidad que se desarrolle, una que piense desde la particularidad de Latinoamérica y Chile o una que trate, por todos los medios, que el desarrollo se asemeje a la idea de modernidad europea?, ¿el pasado, debe tener un fin pedagógico, formador, o simplemente, debe ser comprendido como un desarrollo de hechos que explican el status quo, es decir, una función legitimadora?, fueron las orientadoras en el pensamiento de Lastarria y en el de Bello, al menos, en lo que se circunscribe a la disputa historiográfica.

La especificidad con la cual se alimentó el concepto de lo político estuvo determinada por el contexto particular del siglo XIX latinoamericano; desde este punto de vista, la teoría del Estado, se convierte más bien en una teoría-práctica de la nación. Lo que estuvo en juego no fue sólo una teorización del cómo debe ser la nueva nación, sus ideas, su relación con su pasado, sino, de cómo debe hacerse ésta: su objetivo fue político-fundacional. Esto explica un aspecto que se ha sostenido frecuentemente sobre los pensadores chilenos del siglo XIX: su fuerte inclinación a la praxis. Fueron pensadores de la praxis y no sólo de la teoría abstracta. Sus problemas derivaron de la praxis y su quehacer estuvo determinado por ella y encaminado hacia ella.

Lastarria desde una postura liberal intentó romper con los lazos coloniales y abandonar todo lo que pudo relacionarse con ello ${ }^{17}$, articuló un pensamiento en el cual la historia debió servir para advertir a las nuevas generaciones que no cometieran los mismos errores que sus antecesores; la nación se debía fundar bajo el cuidado de no cometer los errores del pasado, o en otras palabras, la nueva nación debía ser distinta políticamente y socialmente a la del pasado. En definitiva la filosofía de la historia fue una manera de politizar a los jóvenes en busca de poner a la historia bajo algún prisma, y con ello, renegar cualquier intento de imparcialidad.

17 Es necesario mencionar que, a pesar de la veracidad de la frase, el mismo Lastarria se contradijo cuando, en el discurso de 1842 que dictó en la Sociedad Literaria, aceptó y promovió la continuidad de la lengua española, rechazando cualquier tipo de modificación. 
El objetivo por tratar de cambiar las instituciones sociales y con ello, al Estado mismo, se vio reafirmado por las ideas que comenzó a utilizar; la libertad como posesión del derecho, y la correspondencia entre lo que fueron las costumbres de un pueblo civilizado (o en progreso) y sus instituciones, estuvieron bajo el amparo de su funcionalidad práctica. Este objetivo explica a la vez, porque Lastarria no estuvo de acuerdo con la idea de fatalismo así como tampoco con la de Providencia de Herder. Es claro, que tanto Lastarria como Bello reprodujeron, en primera instancia, lo que les fue útil para su proyecto político que tuvo como horizonte la fundación de la nueva nación.

Bello, respecto a su objetivo de lograr un orden y a partir de él hacer progresar a la nación, articuló su visión de la historia como una narración, como una visión imparcial que no se expresó de ninguna forma crítica, en base a ideas filosóficas que sostuvieran el dominio del hombre fuerte sobre el débil y la importancia de la individualización en el estudio para con ello no poner en peligro el desarrollo particular de la nación chilena, y, por último, el eclecticismo que permitió aceptar toda corriente intelectual para generar una convivencia determinada por el orden y la moderación. Esto último, al menos en Andrés Bello, significó el criterio con el cual aceptó, reprodujo y se apropió de las ideas filosóficas europeas; su objetivo práctico estuvo determinado, siempre, por la moderación y el orden, es decir, la fundación de la nación debió estar basada por esas dos características y para ello la relación del pasado debió de ser lo más imparcial posible.

\section{A modo de Conclusión}

Este artículo intenta, en definitiva, aportar un insumo para fundamentar y aceptar la existencia de un pensamiento propiamente latinoamericano. En este sentido, la visión que sostiene que la recepción de las ideas europeas en el siglo XIX derivó sólo a un proceso de reproducción de éstas, pudo ser contradicha al mostrar que tanto Lastarria como Bello al preguntarse cómo debió ser pensado el pasado, terminaron por generar un proceso no de mera reproducción, sino de apropiación ya que sus problemas, basados en el objetivo político fundacional, no existieron en ningún lugar de Europa como para, simplemente, reproducir ideas determinadas bajo ciertas condiciones socio-culturales. Ambos pensadores, en este sentido, debieron conocer ${ }^{18}$ sus propias condiciones y desde allí articular un pensamiento que respondiese por ellas, aun cuando éste tuviera en su formulación vínculos con ideas europeas.

Por otra parte, nos ha interesado mostrar que en la apropiación realizada en el siglo XIX estuvo en juego una concepción política, así como la representación del pasado inmediato; en definitiva, que se estaba poniendo en entredicho la real o no-real fundación de

18 El modo de conocer sí fue distinto en ambos pensadores y el método historiográfico que cada uno utilizó así lo demuestra. Bello quiso conocer, a través de la narración, los hechos del pasado, en cambio, Lastarria quiso conocer el pasado en virtud de ideas preestablecidas para responder si la República independiente cumple los mandatos de la razón (civilización y modernidad) o no. 
una nueva nación en base a directrices de corte liberal o de corte conservador. La disputa historiográfica entre Lastarria y Bello, por ejemplo, no puede ser analizada sólo desde un prisma académico-teórico porque, justamente, en el siglo XIX en Latinoamérica las disciplinas no estuvieron lo suficientemente especializadas para poder conceptualizar ámbitos del saber distintos a los ámbitos propiamente prácticos, como fue el quehacer político.

Por último, el artículo busca poder ser un contenido más para la discusión en torno a la problemática de si ¿existe o no una filosofía chilena o de Chile, con sus características distintivas, su metodología, sus premisas, etc.? Es esta problemática la que ha tenido variadas posturas de diferentes filósofos nacionales, pero lo que creo que falta por desarrollar más, sin menoscabo de investigaciones importantes como los trabajos de Carlos Ruiz y Vasco Castillo, por poner dos ejemplos, es el estudio pormenorizado y regular de aquellas discusiones que se articularon a comienzos de la República. Y esto, porque tengo la sospecha que en ese período se fraguó un modo de articulación entre filosofía y política que no deja de inspirar.*

\section{Bibliografía}

Bello, Andrés, "Investigaciones sobre la Influencia social de la conquista i del sistema colonial de los españoles en Chile, por Don José Victorino Lastarria", en Obras Completas de Don Andrés Bello, Volumen VII, Opúsculos Literarios i Críticos II, Santiago, Impreso por Pedro G. Ramírez, 1884, pp. 71-88.

-, "Bosquejo histórico de la constitución del gobierno de Chile durante el Primer período de la Revolución de 1810 hasta 1814, por Don José Victorino Lastarria", en Obras Completas de Don Andrés Bello, Volumen VII, Opúsculos Literarios i Críticos II, Santiago, Impreso por Pedro G. Ramírez, 1884, pp. 99-106.

, "Modo de escribir la Historia", en Obras Completas de Don Andrés Bello, Volumen VII, Opúsculos Literarios i Críticos II, Santiago, Impreso por Pedro G. Ramírez, 1884, pp. 107-117.

*Artículo recibido: 28 de junio de 2013. Aceptado: 29 de agosto de 2013. 
-------------------, Discurso Inaugural de la Universidad de Chile, 17 de septiembre 1843, Academia Venezolana de la Lengua, Caracas, 1969.

Colmenares, Germán, Las Convenciones contra la cultura: ensayo sobre la historiografia hispanoamericana del siglo XIX, Centro de Investigaciones Diego Barros Arana, Santiago, 2006.

Cordua, Carla, Incursiones, Ediciones Universidad Diego Portales, Santiago, 2007.

DAger, Joseph, "Poner en claro los hechos es escribir la historia: La metodología de la investigación del pasado según Andrés Bello", Pensamientocritico.cl, No 3, 2003. http:// www.pensamientocritico.cl/attachments/089_j-dager-num-3.pdf

Herder, Johann GotTfried, Ideas para una filosofía de la historia de la humanidad, Losada, Buenos Aires, 1959.

Illanes, María Angélica, Chile des-centrado: Formación socio-cultural republicana y transición capitalista (1810 - 1910), Lom, Santiago, 2003.

JAKSIC, IvÁn, Andrés Bello: La pasión por el Orden, Universitaria, Santiago, 2001.

, Academic Rebels in Chile: The Role of Philosophy in Higher Education and Politics, State University of the New York Press, New York, 1989.

JAKSIC, IvÁn y Gracia, J. E., Filosofía e Identidad cultural en América Latina, Monte Ávila Editores, Caracas, 1988.

KempfF, Manfredo, Historia de la filosofía latinoamericana, Zig-Zag, Santiago, 1958.

LASTARria, JosÉ Victorino, "Investigaciones sobre la Influencia social de la conquista i del sistema colonial de los españoles en Chile”, en Miscelánea Histórica i Literaria, Tomo I, Imprenta "La Patria", Valparaíso, 1868, pp. 3- 138.

, "Bosquejo histórico de la constitución del gobierno de Chile durante el Primer período de la Revolución de 1810 hasta 1814”, Miscelánea Histórica i Literaria, Tomo I, Imprenta "La Patria”, Valparaíso, 1868, pp. 139-268.

de Educación Pública, México, 1944.

, "Elementos de Sociología", Ediciones de la Secretaría , Recuerdos Literarios, Zig-Zag, Santiago, 1968. 

rria a una Sociedad de Literatura de Santiago, en la sesión del tres de mayo de 1842, Impr. De M. Rivadeneyra, Valparaíso, p. 2842.

Oyarzún, Luis, El Pensamiento de Lastarria, Editorial Jurídica de Chile, Valparaíso, 1953.

Salazar Bondy, Augusto, ¿Existe una filosofía de nuestra América?, Siglo Veintiuno, México, 1969.

Santos, José, Conflicto de Representaciones, FCE, Santiago, 2010.

Stuven, Ana María, La Seducción de un Orden: las elites y la construcción de Chile en las polémicas culturales y políticas del siglo XIX, Ediciones Universidad Católica de Chile, Santiago, 2000.

Subercaseaux, Bernardo, Cultura y sociedad liberal en el siglo XIX (Lastarria, ideología y literatura), Editorial Aconcagua, Chile, 1981.

go intercultural". Dialogosfelafacs.net. http://www.dialogosfelafacs.net/dialogos_epoca/ pdf/23-BernardoSubercaseaux.pdf

ZeA, Leopoldo, El pensamiento Latinoamericano, Ariel, Barcelona, 1976. 